\title{
Intraductal papillary mucinous neoplasm of the pancreas - when and how to operate?
}

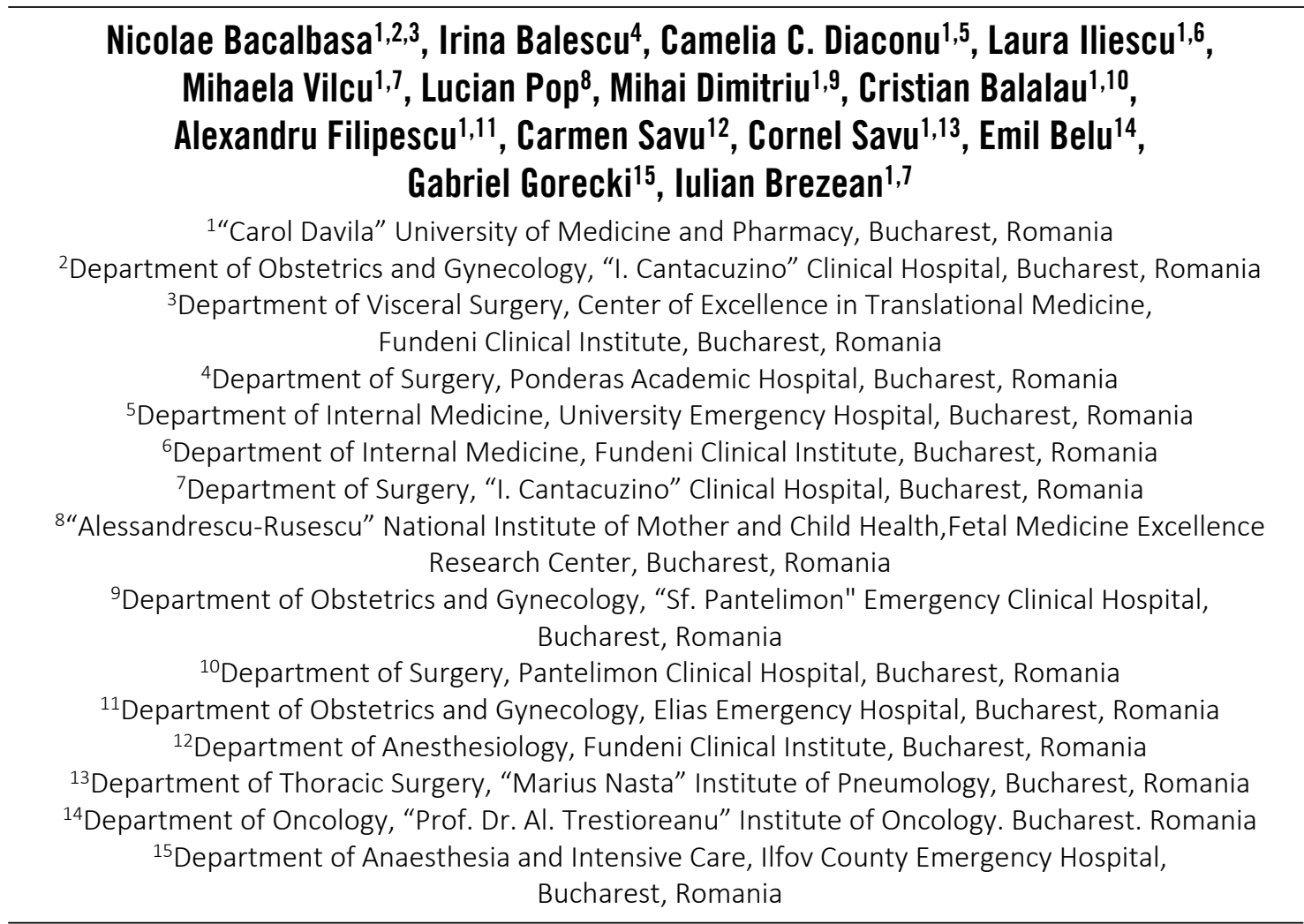

\begin{abstract}
Described for the first time in 1982, intraductal papillary mucinous neoplasms have been widely studied so far. According to the site of origin and location, it seems that these tumors have a specific risk of malignisation and therefore their type of treatment as well as the moment in which it should be applied should be carefully tailored. In this respect, multiple therapeutic strategies have been proposed, ranging from follow up and limited excision to total pancreatectomy. The current paper is a literature review of the largest studies conducted on the theme of the therapeutic strategies in intraductal papillary mucinous neoplasms.
\end{abstract}

Keywords: intraductal papillary mucinous neoplasm, main duct, branch duct, resection

\section{INTRODUCTION}

Intraductal papillary mucinous neoplasms (IPMN) of the pancreas represent intraductal, mucin producing, cystic lesions with a broad spectrum of histopathological aspects ranging from benign to malignant lesions $[1,2]$. Cases presenting benign lesions are associated with an excellent long term outcome [3]. When it comes to patients presenting
IPMN with foci of carcinoma in situ, the natural evolution might conduct to the development of invasive pancreatic adenocarcinoma [3]. In such cases, the prognostic becomes significantly poorer, similar to the one reported in invasive pancreatic adenocarcinoma [3]. Described for the first time in 1982 by Ohashi, this pathologic entity reported increasing incidence in the last decades especially in elderly patients $[4,5]$. 


\section{HISTOPATHOLOGICAL ASPECTS}

Almost two decades later, Tanaka et al. published a consensus guideline which classified these lesions into main duct, branch duct and mixed type tumors; interestingly, it seems that the location of these tumors and their origin also influences the histopathological subtype, a higher rate of invasive and carcinoma in situ areas being revealed in main duct tumors (MDT) when compared to branch duct (BDT) ones [6]. Moreover, in up to $80 \%$ of cases with IPMN multiple lesions might develop synchronously or metachronously [7]. In this respect, the therapeutic strategy as well as the type of surgical resection has been widely debated so far. This aspect is mainly justified by the fact that in a certain number of cases the preoperative diagnostic of malignancy cannot be established; in this respect, it has been stated that all cases presenting MDT and cases presenting symptomatic, larger than $3 \mathrm{~cm}$ BDT in association with those who present mural nodules should be routinely submitted to surgery even in the absence of a histopathological diagnostic of malignancy [8]. As for their outcomes, it has been demonstrated that while patients presenting benign features report an excellent long term outcome, cases presenting malignant transformation have poorer outcomes, comparable with cases diagnosed with pancreatic adenocarcinoma $[9,10]$.

\section{SURGICAL STRATEGIES IN IPMN PATIENTS}

In order to improve the long term outcomes of patients diagnosed with IPMN and to minimize the long term morbidity, multiple surgical strategies have been proposed; therefore, due to the fact that a high number of cases submitted to surgery for IPMN will present positive resection margins or will develop recurrent disease at a point of their evolution, certain authors proposed total pancreatectomy as the option of choice. However, it should not be omitted the fact that in such cases significant comorbid conditions such as permanent insulin dependence will develop. Meanwhile, the absence of exocrine pancreatic function will transform the patient into a permanent consumer of supplements of pancreatic enzymes [11].

Moreover, a study published on the issue of partial pancreatectomy versus total pancreatectomy by the Chinese authors conducted by Wang et al. failed to demonstrate any improvement in terms of cancer specific survival and respectively overall survival among patients submitted to total pancreatectomy versus pancreatoduodenectomy [8].
When it comes to the importance of the status of surgical margins after resection of IPMN, the Japanese authors conducted by Yamaguchi et al. published a study in which they included 55 cases of IPMN submitted to surgery; among these cases the authors underlined the fact that positive margins were present in 16 cases, this positivity not being significantly correlated with the risk of recurrence; however, cases presenting unifocal lesions had a significantly higher risk of developing recurrent disease when positive margins were encountered while this correlation failed to be a significant one in cases presenting multifocal lesions. Meanwhile, the authors underlined the fact that positive resection margins were significantly correlated with the presence of multifocal IPMN [3].

One of the first consensus conferences held on the theme of surgical indications in BDT was the one in Sendai in 2006 and stated that cases presenting larger than $3 \mathrm{~cm}$ cysts as well as those who present mural nodules, clinical symptoms, positive cytology and dilatation of the Wirsung duct should be considered as candidates for surgery even if a single criterion from the above mentioned list is present [6]. Six years later, a novel consensus conference was released in Fukuoka; according to this consensus BDT should be classified in two groups: the first group included cases considered to have worrisome features such as larger than $3 \mathrm{~cm}$ cysts, enhanced and thickened cystic walls, a long personal history of pancreatitis, a main pancreatic duct measuring between 5-9 mm or a significant modification in regard of the main's duct calibre and distal atrophy as well as those presenting non enhanced lymphadenopathy while the second group should include patients considered to be at high risk, referring to cases presenting jaundice, a larger than 10 $\mathrm{mm}$ Wirsung duct or enhanced solid components. Depending on the presence of these features, cases in the first category could be submitted to close surveillance while those in the second category should be submitted to surgery [12].

Further studies came to demonstrate that Fukuoka consensus criteria brought a considerable improvement in order to increase the sensibility of identifying patients who could benefit most from surgery; however, the specificity remained poor. Therefore, in a meta-analysis conducted by Heckler et al. and published in 2017 the author included 15 studies and 2,710 patients and demonstrated that, when it came to Fukuoka's criteria, they were associated with a sensibility of $83 \%$ and with a specificity of only $53 \%$. In this respect the authors considered that although Fukuoka consensus 
brought a significant improvement in the management of BDT, a significant number of cases are still over-treated and therefore new criteria should be developed in order to avoid surgical over-therapy [13].

One of the most representative studies which was also transformed into an European consensus was published in 2018 and stated that all cases presenting symptomatic lesions as well as suggestive imagistic criteria (intramural nodules, solid masses, a main pancreatic duct larger than $10 \mathrm{~mm}$ ) should be submitted to surgery; meanwhile cases diagnosed with two of the following features: grow rate $>5 \mathrm{~mm} /$ year, serum levels of CA 19-9>37 $\mathrm{U} / \mathrm{ml}$, main pancreatic duct measuring between 5 and $9.9 \mathrm{~mm}$, new onset of diabetes, larger than 4 $\mathrm{cm}$ cysts and larger than $5 \mathrm{~mm}$ contrast enhancing mural nodules present relative indications for surgery. In the meantime cases presenting a single criterion from the above mentioned list are suitable for close imagistic surveillance by magnetic resonance or endoscopic ultrasound [14].

\section{CONCLUSIONS}

IPMN represent a relatively novel category of pancreatic lesions with broad spectrum of histo-

\section{REFERENCES}

1. Shi $\mathrm{C}$, Hruban RH. Intraductal papillary mucinous neoplasm. Hum Pathol. 2012;43:1-16.

2. Niedergethmann $M$, Grutzmann $R$, Hildenbrand $R$ et al. Outcome of invasive and noninvasive intraductal papillary-mucinous neoplasms of the pancreas (IPMN): A 10-year experience. World J Surg. 2008;32:2253-60.

3. Yamaguchi J, Kaneoka $Y$, Maeda A et al. Positive surgical margins in surgically treated unifocal and multifocal IPMN. Int J Surg. 2016;28:51-55

4. Ohashi K, Murakami Y, Maruyama M. Four cases of mucous secreting pancreatic cancer. Prog Diagn Endosc 1982;20:348-351.

5. Khan S, Sclabas G, Reid-Lombardo KM. Population-based epidemiology, risk factors and screening of intraductal papillary mucinous neoplasm patients. World J Gastrointest Surg. 2010;2:314-318.

6. Tanaka M, Chari S, Adsay V et al. International consensus guidelines for management of intraductal papillary mucinous neoplasms and mucinous cystic neoplasms of the pancreas. Pancreatology. 2006;6:17-32.

7. Matthaei H, Norris AL, Tsiatis AC et al. Clinicopathological characteristics and molecular analyses of multifocal intraductal papillary mucinous neoplasms of the pancreas. Ann Surg. 2012;255:326-333.

8. Wang $X$, Hong $X$, Pang $\mathrm{H}$ et al. Selecting optimal surgical procedures for intraductal papillary mucinous neoplasm (IPMN): An analysis pathological aspects ranging from benign to malignant features. While in cases presenting main duct origin it is widely accepted that surgery should be the option of choice, in cases originating from the branch duct things have been submitted to permanent changes, different consensus guidelines being proposed so far regarding the moment in which surgery should be performed. As for the type of procedure, while in certain cases total pancreatectomy has been proposed due to the multifocal features of these lesions, certain authors came to demonstrate that this might be an exaggerated option while severe long term complications might be induced; meanwhile, a clear difference in terms of survival failed to be demonstrated between cases submitted per se to total pancreatectomy and those submitted to less extended surgical procedures.

\section{Acknowledgements}

This work was supported by the project entitled „Multidisciplinary Consortium for Supporting the Research Skills in Diagnosing, Treating and Identifying Predictive Factors of Malignant Gynecologic Disorders", project number PN-III-P1-1.2-PCCDI2017-0833.

Conflict of interest: none declared Financial support: none declared

based on the Surveillance, Epidemiology, and End Result registry database. Eur J Surg Oncol. 2016;42:1526-32.

9. Frankel TL, LaFemina J, Bamboat ZM et al. Dysplasia at the surgical margin is associated with recurrence after resection of non-invasive intraductal papillary mucinous neoplasms. HPB (Oxford) 2013; 15:814-21.

10. White R, D'Angelica M, Katabi $\mathrm{N}$ et al. Fate of the remnant pancreas after resection of noninvasive intraductal papillary mucinous neoplasm. J Am Coll Surg. 2007;204:987-993.

11. He J, Cameron JL, Ahuja $\mathrm{N}$ et al. Is it necessary to follow patients after resection of a benign pancreatic intraductal papillary mucinous neoplasm? J Am Coll Surg. 2013;216:657-665.

12. Tanaka M, Fernandez-del Castillo C, Adsay V et al. International consensus guidelines 2012 for the management of IPMN and MCN of the pancreas. Pancreatology. 2012;12:183-197.

13. Heckler M, Michalski CW, Schaefle S et al. The Sendai and Fukuoka consensus criteria for the management of branch duct IPMN - A meta-analysis on their accuracy. Pancreatology. 2017;17:255-262.

14. European evidence-based guidelines on pancreatic cystic neoplasms. Gut 2018;67:789-804. 